\title{
Issues on Grid Convergence
}

\author{
T. Wang, Q. S. Guo \\ Academy of Armored Force Engineering, Beijing 100072, China \\ Email: godbless126@126.com
}

\begin{abstract}
In the numerical simulation of flow fields, problems exist in controling the grid spacing and balancing the contradiction between the number of grid and calculation error. Two issues which affect grid convergence have been investigated in the current paper.

The first issue is critical grid spacing. It would be more helpful if the grid spacing can be reasonably forecasted before calculation. In the aspect of numerical calculation, it is affected by the order of differential formula. In the aspect of property of fluid, it is concerned with the Reynolds number of flow field.

The second issue is distribution characteristics of grid. Several factors were investigated to analyze the impact of distribution characteristics of non-uniform grid, including uniformity, length-to-width ratio and orthogonality on the computational convergence.

In addition, the error index on grid convergence is also important. Although higher order scheme was said to be more reliable, larger error comparing to low order scheme might be found. It is needed to weigh schemes through a consistent index. Referring to Roache's GCI (Grid Convergence Index), an approach for high dimension and mix-order problems is employed to measure the grid convergence. 2 -D flows around square cylinder was used to verify above arguments. The Reynolds number is 22000 , turbulence intensity is $2 \%$, and QUICK scheme was used for convection term. To estimate the influence of grid spacing, three levels of grid were chosen. RANS equations, RNG $k-\varepsilon$ turbulence model, PISO algorithm was used. The figures of vorticities show that all three levels of grid can capture the vorticity separation. However, the coarsest grid can not distinguish the vorticity street, the finer grid can distinguish the street but not the boundary between vorticities because of coarser grid in field far from cylinder, the finest grid can distinguish both the street and the boundary.

The results indicate that the order of difference scheme and grid spacing affect calculation considerably. Uniformity and length-to-width ratio nearly do not affect calculation in acceptable range. Therefore, partial adaptation is a good choice for higher precision and efficiency. At last, a general process for estimation of the grid convergence as a guideline for grid generation is concluded.
\end{abstract}

\section{REFERENCES}

1. Sjogreen B, Yee H C. Grid convergence of high order methods for multiscale complex unsteady viscous compressible flows. J Comput Phys, 2003; 185: 1-26

2. Faragher J. Probabilistic methods for the quantification of uncertainty and error in computational fluid dynamics simulations. DSTO-TR-1633, 2004

3. Zhang H X, Guo C, Zhong W G. Problems about grid and high order schemes. J Mechanics, 1999; 31: 398-405 (in Chinese) 